\section{Den Hauptverursacher dingfest machen}

$F^{u}$ ür die meisten Allergologen ist die Diagnostik mit Allergenkomponenten zunächst sehr verwirrend. Das liegt mitunter daran, dass sie sich seit der Entdeckung von Immunglobulin E 1966 an die Diagnostik mit Gesamtextrakten gewöhnt haben. Warum sich eine Umstellung dennoch lohnen könnte, erklärt Prof. Dr. Thilo Jakob, Freiburg.

\section{? Herr Professor Jakob, welche Vorteile bie-} ten Immuno CAP $^{\circledR}$ Allergenkomponenten gegenüber der Testung auf Gesamtextrakte? Jakob: Die Allergiediagnostik mit Allergenkomponenten ermöglicht eine gezielte Testung auf relevante Sensibilisierungen gegenüber den allergieauslösenden Komponenten. In der konventionellen Allergiediagnostik, die Allergenextrakte einsetzt, verwenden wir komplexe Gemische aus einer Vielzahl von Proteinen. Hiervon sind als allergieauslösende Moleküle häufig nur einzelne Proteine wirklich relevant. Die Erkenntnisse der Molekularen Allergologie haben es uns in den letzten Jahren ermöglicht, statt der Extrakte nun Einzelproteine für die Allergiediagnostik einzusetzen. Die Verwendung dieser Allergenkomponenten erlaubt eine höhere analytische diagnostische Empfindlichkeit und Präzision. Zusätzlich ermöglicht sie in vielen Fällen eine Abgrenzung von Primärsensibilisierungen und Kreuzsensibilisierungen.

? Wieso ist die klare Unterscheidung zwischen Primärsensibilisierung und Kreuzreaktion für den Behandlungserfolg eines Allergikers wichtig?

Jakob: Die Unterscheidung zwischen Primärsensibilisierung und Kreuzreaktion ist vor allem für die Indikationsstellung der Immuntherapie von essenzieller Bedeutung. Die Wirksamkeit der spezifischen Immuntherapie ist dann am größten, wenn der primär sensibilisierende Allergenextrakt eingesetzt wird. Um einen möglichst hohen Nutzen der spezifischen Immuntherapie zu erreichen, sollte die Therapie mit dem „Hauptverursacher" und nicht mit „Mittätern“ erfolgen und genau diese Abgrenzung wird durch die verbesserte Diagnostik mit Allergenkomponenten ermöglicht.

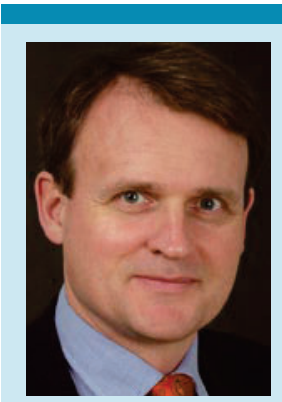

„Die komponentenbasierte Diagnostik erlaubt die Erfassung komplexer Sensibilisierungsmuster bei polyvalenter Allergie.“

Prof. Dr. Thilo Jakob

Abteilung Allergologie und Immundermatologie und Forschergruppe Allergologie, Universitäts-Hautklinik Freiburg

? Welche Bedeutung haben die innovativen Bluttests speziell für Risikopatienten? Jakob: Besonders im Bereich der Nahrungsmittelallergie ermöglicht die komponentenbasierte Allergiediagnostik eine bessere Information über das relative Risiko des Patienten auf bestimmte Allergene mit einem schweren, zum Teil lebensbedrohlichen Allergieschock zu reagieren. Nehmen wir das Beispiel Erdnussallergie: Der Großteil der Patienten, die im extraktbasierten Allergietest positiv auf Erdnuss reagieren, sind Birkenpollenallergiker, die eine Kreuzreaktion auf das Homolog des Birkenpollenallergens Bet v 1 in der Erdnuss - Ara h 8 zeigen. Das Bet-v-1-Homolog Ara h 8 ist ein PR10-Protein und ist hitze- und verdauungssensibel, so dass es in der Regel maximal ein orales Allergiesyndrom auslöst. Im Gegensatz hierzu sind andere Allergene der Erdnuss, z. B. die Speicherproteine Ara h 1, Ara h 2, Ara h 3 oder das Lipidtransferprotein Ara h 9, hitze- und verdauungsresistent und häufiger Auslöser von schweren systemischen Reaktionen. Im Gegensatz zur extraktbasierten Diagnostik kann die komponentenaufgelöste Diagnostik nun Patienten mit ernst zu nehmender Erdnusssensibilisierung von den Patienten abgrenzen, die mit dem birkenpollenhomologen Allergen kreuzreagieren und damit ein geringes Risiko einer systemischen Reaktion nach Erdnussgenuss haben.

red

Nach Informationen von Phadia, Freiburg

\section{Allergieratgeber für Eltern}

Wie können Eltern ihren allergiekranken Kindern helfen? Welche Therapiemöglichkeiten existieren und wie wirksam ist die „Allergie-Impfung"? Antworten auf diese Fragen verspricht der Ratgeber: „Allergie bei Kindern - Tipps und Hinweise für Eltern allergischer Kinder" von ALK-Abelló, einem Hersteller von Präparaten für die spezifische Immuntherapie. Die handliche Broschüre informiert über die Ursachen der Allergie, Forschungsergebnisse und Therapiemethoden wie die Hyposensibilisierung. In der Broschüre findet sich überdies eine Checkliste „Reagiert Ihr Kind allergisch?“, ein Pollenflugkalender sowie eine Auflistung der wichtigsten AllergiePatienten-Organisationen. Unter www. alk-abello.com/de/patienten/patientenbroschüren kann der Ratgeber kostenlos heruntergeladen werden.

Nach Informationen von ALK-Abelló, Wedel

\section{Pollenflug auf dem Android}

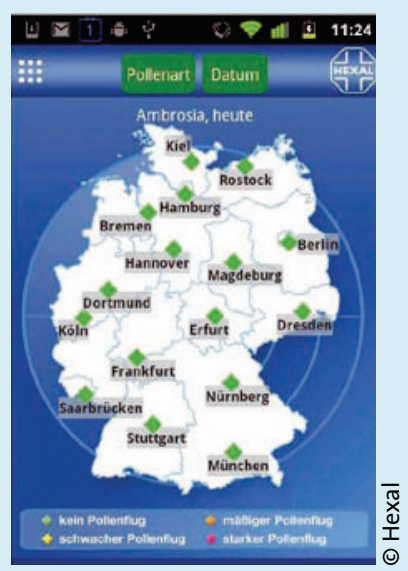

Damit Pollenallergiekranke sich unterwegs immer aktuell über den Pollenflug informieren können, bietet das Pharmaunternehmen Hexal die App „PollenflugVorhersage“ nun auch für Android-Handys unter http://market.android.com zum kostenlosen Herunterladen an. Neben einer Drei-Tages-Vorhersage für 14 Pollenarten für jeden Ort in Deutschland können auch die Pollenflug-Trends abgefragt werden. Die Daten werden dreimal täglich aktualisiert. Wer viel unterwegs ist, kann die Ortserkennung einfach über GPS aktivieren. Alternativ gibt der Nutzer einmalig die für ihn wichtigsten Postleitzahlen und Auslöser ein und erhält daraufhin seine individuelle Pollen-Vorhersage. red

Nach Informationen von Hexal, Holzkirchen 